\title{
Iris Feature Extraction Algorithm valuation Based on the Fuzzy Integral Evaluation Model
}

\author{
Liu Jin $^{1, \text { a }}$, Liu Changming ${ }^{1, a}$, Sun Yanjun ${ }^{1, \text { a }}$ \\ ${ }^{1}$ Computer Department, Air Force Aviation University, changchun 130022, China \\ aemail: mka1982@163.com
}

Keywords: Iris Identification; Fuzzy Integral Model; Feature Extraction; Affine

\begin{abstract}
Iris Feature Extraction Algorithm Evaluation is an important link of evaluation on iris identification system. For the need of optimal feature extraction algorithm according to the identification, this thesis provides an evaluation scheme based on the fuzzy integral model, and builds a relevant evaluation model, which proves to make a scientific evaluation on the algorithm in the phase of feature extraction.
\end{abstract}

\section{Introduction}

Various identification algorithms emerge in endlessly subject to the vigorous development of iris recognition technology. However, it is mainly focused on the study of core algorithm, with the comparison rarely involved of advantages and disadvantages between those algorithms. The credibility of the analysis and application results are lowered to different extents by such issues as the feasibility of those algorithms and the suitable fields of the identification characteristics of those algorithms, which requires to establish such an evaluation model adapted with the iris recognition algorithms[1-4] as to evaluate the recognition effects, thus making the comparison between various algorithms objective and accurate, and providing a scientific and reasonable quality standard for iris recognition to make the recognition system of the optimum allocation. In addition, it is effective and practical to establish such an evaluation model based on the Fuzzy Integral [4] in fuzzy mathematics.

Widely applied as it is, Fuzzy Integral Model has no requirement on the additively of weights and may describe the interaction between evaluation indexes with fuzzy measure, which not only reflects the importance of all evaluation indexes in the system, but also embodies the influence of their interactions on the system.

When evaluating the biometric recognition system, the Fuzzy Integral Model will show its obvious advantages over dealing with various uncertain information and conducting comprehensive evaluation and comparison of recognition algorithms. The evaluation results if obtained by Fuzzy Integral Model are so responsive to the change of indicators that even a slight change of important indexes will be reflected, thus making the conclusions more objective. It is quite suitable to rationally select algorithms as per the recognition requirements, especially for the quality assessment of biometric recognition algorithms.

\section{Algorithm Design of Assessment Model Based on Fuzzy Integral}

The theory essence of Surgeon Fuzzy Integral is quoted in the Fuzzy Integral herein, which is a new concept different from the classical integral with "Large or Small $(\vee-\wedge)$ ") as its algorithm. One of the differences between Fuzzy Integral Model and Fuzzy Comprehensive Evaluation Model is that the former has no remark set and only calculates the membership degree to "the optimal". Therefore, the optimum value of each factor to "the optimal" shall be determined in addition to the factor set and weight set. 


\section{Fuzzy Integral may be used to construct the comprehensive evaluation model.}

Given that domain of discourse $U=\left(u_{1}, u_{2}, \cdots \cdots u_{n}\right)$ contains all factors to be considered in the comprehensive evaluation, $\Omega(\mathrm{U})$ is the backup domain, $\Gamma$ is the possibility measure on $\Omega$, and the weight set is $M=\left(m_{1}, m_{2}, \cdots \cdots m_{n}\right)$. If the status of an evaluation object on the factor set is $G=\left(g_{1}, g_{2}, \cdots \cdots g_{n}\right), G \in F(U)$, then its evaluation may be expressed as:

$$
\int_{-U} g(x) \times \Gamma(\bullet)=G \times M=\stackrel{n}{\vee}\left(g_{i} \wedge m_{i}\right)
$$

Wherein, different from the classical integral, $\vee$ and $\wedge$ indicates "big” and "small".

\section{Design procedures to use Fuzzy Integral Model}

Determination of evaluation object: Select the algorithm to evaluate and denote it as $F=\left(F_{1}, F_{2}, \cdots \cdots F_{k}\right)$. Make an evaluation conclusions conforming to the recognition requirements by contrasting the elements in the set, and select the best algorithm as per the conclusion, thus realizing the optimal configuration of recognition algorithm.

Determination of factor set: Given that $U=\left(u_{1}, u_{2}, \cdots \cdots u_{n}\right)$ is the set of $\mathrm{n}$ kinds of elements, which contains all factors to be considered in the fuzzy integral evaluation, referred to as the factor set, it will be the domain of discourse.

Extraction and affine of data in factor set: Extraction and affine of data in factor set are a key to the evaluation with Fuzzy Integral Model. Extract and quantify the attributes having important impact on the recognition results, which will be then affined to the Fuzzy Integral Model for the evaluation detection.

Determination of weight set: The emphasis degree of each factor or the importance of each factor against the system may vary with the evaluation requirements. Therefore, weight set $A=\left(a_{1}, a_{2}, \cdots \cdots a_{n}\right)$ is adopted as the allocation weights for $\mathrm{n}$ kinds of factors; in other words, the importance of elements in the factor set is determined.

Determination of the optimum value of each factor: The optimum value of a factor is the value determined according to certain standards to make the factor in its optimal state, the selection of which is the precondition to determine a single evaluation vector. The optimum value shall be determined by summarizing a large number of experimental results. $V=\left(v_{1}, v_{2}, \cdots \cdots v_{n}\right)$

\section{Extraction and Affine of Parameters in Feature Extraction Algorithm}

Recognition rate: Recognition rate is used to indicate the identification accuracy, namely the percentage of the correct recognitions against the total identifications. If the affine recognition rate is indicated by $U_{1}$, the number of correct recognitions is indicated by $N_{\text {True }}$ and the number of total recognitions is indicated by $\mathrm{N}$ after feature extraction, then:

$$
U_{1}=\frac{N_{\text {True }}}{N}
$$

False rejection rate: If the affine false rejection rate is indicated by $U_{2}$, the number of false rejections is indicated by $N_{F R}$ and the number of total recognitions is indicated by $\mathrm{N}$ after feature extraction, then:

$$
U_{2}=\frac{N_{F R}}{N}
$$

False acceptance rate: If the affine false acceptance rate is indicated by $U_{3}$, the number of false acceptances is indicated by $N_{F A}$ and the number of total recognitions is indicated by $\mathrm{N}$ after feature 
extraction, then:

$$
U_{3}=\frac{N_{F A}}{N}
$$

Processing time: Processing time is indicated by $U_{4}$. It is just as simple as to set a timer to record the processing time from the start to the end of the algorithm.

Influence proportion on iris feature matching: The success rate of feature matching is determined by whether the iris image feature is extracted effectively, and the influence proportion is reflected in the influence degree on recognition rate. According to the feedback information from the previous experimental studies, the difference between recognition rates is usually in the range of $1 \% \sim 5 \%$ by comparing the extraction algorithms of different features. If the recognition rate of such algorithm is increased by over $4 \%$, it will be deemed that it has great influence on iris feature matching; if by $2 \% \sim 4 \%$, the influence is not obvious; if by $1 \% \sim 2 \%$, the influence is small; if by less than $1 \%$, it may be deemed that there is no influence.

Given that $U_{5}$ is the affine of $Q_{5}$ in the fuzzy comprehensive evaluation model, then:

$$
U_{5}=\{0,1,2,3\}
$$

If iris feature extraction has no influence on feature matching, namely invalid, $U_{5}=0$; if there is small influence, $U_{5}=1$; if the influence is not obvious, $U_{5}=2$; if the influence is great, $U_{5}=$ 3.

\section{Analysis of Evaluation Results by Feature Extraction Algorithm Based on Fuzzy Integral Assessment Model Conclusion}

Among the feature extraction algorithms of maxima of the wavelet modulus, wavelet zero-crossing and qualification of wavelet multi-scale features, if take into account of the effect on false rejection rate and influence proportion on iris feature matching, the qualification of wavelet multi-scale features is the best, followed by maxima of the wavelet modulus and then wavelet zero-crossing, with the differences in membership degree to "the optimal" of $0.087,0$ and 0.041 respectively. Therefore, it can be concluded that subject to the weight distribution under this evaluation, the performances of the three approximate into an arithmetic progression. However, the assessment result is not absolute, which may vary with the distribution weights caused by different focuses. The Fuzzy Integral Model is superior in that it allows the users to compare various algorithms by different focuses and interactions between all factors.

\section{Conclusions}

Fuzzy Mathematics Model is widely applied in the practice, especially making the evaluation results more objective and scientific, which is well verified by the instances herein. Fuzzy integral evaluation is just one method of fuzzy mathematics, and there are still many other scientific methods to be further explored to reasonably evaluate the recognition algorithms. Necessary and complex to as it is, the evaluation of iris recognition effect requires more attention and participation from various research institutions and enterprises.

\section{References}

[1] Hatem N N. Automated Instrumentation Evaluation and Diagnostics of Automatic Taget Recognition Systims[A]. In: SPIE Automatic Object Recognition Conference[C], 1990, (7): 202-213.

[2] Zhuang Zhaowen, Li Xiang, Li Yanpeng, Wang Hongqiang. Effect Evaluation Technology of Automatic Target Recognition[M]. National Defence Industry Press, 2006.10, 132-138 158-163. 
[3] Li Yanpeng, Shi Fuzhong, Li Xiang, Zhuang Zhao. Effect Evaluation of Target Recognition Based on Fuzzy Comprehensive Assessment[J]. Application Research Of Computers, 2005, (3): $1-5$.

[4] Li Yanpengi, Li Xiang, Zhuang Zhaowen. Effect Evaluation of Target Recognition Based on Sugeno Fuzzy Integral[J]. Journal of System Simulation, 2005, (5): 235-254.

[5] Zhang Wei. Framework Design and Implementation of Object-oriented Hierarchical and Open Iris Recognition System[D]. Master's Thesis, Jilin University, 2006.3:1-96.

[6] Boles W.W. Security system based on human iris identification using wavelet transform[J]. Engineering Applications of Artificial Intelligence, 1998, 11(1): 77-85.

[7] Boles W.W. Security system based on human iris identification using wavelettransform[J]. Adelaide, Aust: IEEE, Piscataway, NJ, USA, 1997: 533-541.

[8] Ming Xing. Research on Iris Texture Coding and Matching in Iris Recognition Technology [D]. Master's Thesis, Jilin University, 2002.3:1-130.

[9] S. Pittner, S.V. Kamarthi. Feature Extraction from Wavelet Coefficients for Pattern Recognition Tasks[J]. IEEE Transactions on Pattern Analysis and Machine Intelligence, 1999, 21(1): 83-88.

[10] Shinyoung Lim, Kwanyong Lee, Okhwan Byeon, Taiyun Kim. Effective Iris Recognition System by Optimized Feature Vectors and Classifie[J]. Lecture Notes in Computer Science, 2008: 348-357.

[11] Zhu Xiaodong, Ming Xing, Liu Jing, Iris Recognition with Binary Encoding Based on Quasi-zerotree Wavelet, Proceedings of the 6th International Progress on Wavelat analysis and Active Meia Technology, 2005, (1): 432-437. 